\title{
Perceptions of Undergraduate Engineering Students about Learning Objectives of Undergraduate Laboratories
}

\author{
Kimia Moozeh $^{1,2}$, Deborah Tihanyi ${ }^{3}$, Jennifer Farmer ${ }^{1}$, and Greg Evans, ${ }^{1,2,4}$ \\ ${ }^{1}$ Dept. of Chemical Engineering and Applied Chemistry, ${ }^{2}$ Collaborative Specialization in Engineering Education \\ ${ }^{3}$ Engineering Communication Program, ${ }^{4}$ Institute for Leadership Education in Engineering \\ University of Toronto \\ kimia.moozeh@mail.utoronto.ca
}

\begin{abstract}
The paper presents student perceptions about learning objectives of laboratories. Three focus group sessions were conducted with chemical engineering undergraduate students at UofT as part of a larger project to enhance the learning outcomes of laboratories.

In this study, thirteen laboratory learning objectives developed at the ABET colloquy in 2002, were used as a framework to determine the strengths and limitations of the laboratories. These learning objectives cover cognition, psychomotor and affective domains of knowledge.

The results indicate that improvements are needed with respect to providing opportunities for students to be creative, devise their own procedures, repeat experiments and improve communication skills. In addition, ethics in the lab and safety need more emphasis.
\end{abstract}

Keywords: chemical undergraduate laboratories, ABET learning objectives, laboratory-based learning, virtual and remote labs.

\section{INTRODUCTION}

Undergraduate engineering laboratories provide an opportunity for students to practice skills such as problem solving, analytical thinking, and hands on skills $[13,14,17]$. Moreover, labs have the potential to motivate students [5]; foster an understanding of scientific inquiry; promote professional, social and teamwork skills [17], and teach students about the power and limitations of theories [4].

Another factor contributing to the importance of laboratories in engineering education is the shift towards constructivist pedagogy [14]. According to this theory, knowledge is not simply transferred from one person to another [3]. Rather, individuals actively learn by constructing their own meaning through experience and interaction with their environment [9]. Undergraduate laboratories are appropriate environments for such experiential learning [3].
However, despite the importance of laboratories in engineering curricula $[1,7,8]$, there is a general agreement that they result in a poor return in terms of learning outcomes when the time, effort and money invested in them are considered [1]. A research project is currently being conducted to enhance the learning experience of students in engineering laboratories by developing digital learning objects (DLOs), and integrating them with hands-on laboratory experiments based on Kolb's cycle.

As the first step, student perceptions regarding their laboratory-based learning experiences were sought through surveys and focus groups. The main purpose of the study was to identify gaps in the laboratories, and address them through DLOs. This paper focuses on the results obtained from the focus groups.

Thirteen laboratory learning objectives were used as a framework in our focus groups to determine the strengths and limitations of the laboratories. In an effort to determine the fundamental objectives of undergraduate instructional laboratories, the Accreditation Board for Engineering and Technology (ABET), held a colloquy in 2002. Thirteen laboratory learning objectives were developed as the result of this colloquy. Clear learning objectives could serve as a framework and benchmark to develop and assess laboratories [12]. The objectives define what students should be able to do after completing the undergraduate engineering lab, and are as follows (The definitions are in Appendix 1):

1.Instrumentation 2.Models 3.Experiment 4.Data Analysis 5. Design 6. Learn From Failure 7. Creativity 8. Psychomotor 9. Safety 10. Communication 11. Teamwork 12 Ethics in the Lab 13. Sensory Awareness.

This paper presents the perceptions of undergraduate chemical engineering students at the University of Toronto (UofT), St. George campus about their laboratory-based experiences, using these learning objectives as a framework.

\section{METHODOLOGY}

The study protocol for the focus groups was approved by the UofT research ethics board. Participants $(n=10)$ 
were volunteer undergraduate chemical engineering students in their second to fourth year of study. The female:male ratio was four:six. The students were divided based on their year of study, and three focus group sessions were conducted. The sessions were conducted in the middle of the second semester to ensure second-year students had experienced at least one semester of practical work.

The mandatory second-year chemical engineering laboratory course at UofT focuses on applied chemistry laboratory techniques in the area of analytical, inorganic and organic chemistry. The course is both analytical and synthetic in nature- students investigate chemical phenomena by probing the nature of the chemical sample provided to them, as well as synthesize inorganic and organic chemicals using established processes and laboratory techniques. Elements of process and industrial chemistry and practice (including Green Chemistry) are also explored. Third-year students have to take three laboratory courses: A Thermodynamics/Kinetics laboratory, a Processes Design laboratory, and Separation Processes laboratory. The latter two are situated in the Chemical Engineering Unit Operation laboratory. Fourthyear students don't have to take any mandatory lab courses, and hence the participants were reflecting on their past experiences. These laboratory courses are meant to be a practical supplement to the learning experiences of some second- and third-year lecture-based courses.

Students were asked to discuss topics such as pre-labs, laboratory reports, real-world relevance of laboratories as well as the thirteen objectives identified by the colloquy. Each learning objective's definition was written on a card, with an example to clarify the definitions. The students were asked to discuss these objectives, and to comment whether the laboratories had helped them learn and improve these skills. The focus group sessions were audio recorded, transcribed and analyzed.

\section{RESULTS}

The responses were analyzed to determine how student opinions change as they progress through the curriculum, and are categorized into three groups:

Cluster 1. There was a consensus among participants regarding psychomotor, sensory awareness, and models learning objectives, as students indicated laboratories had helped them improve these skills.

Cluster 2. There was no consensus among participants regarding teamwork, safety, instrumentation, ethics in the lab, learn from failure and data analysis. In other words, the responses regarding these learning objectives differed depending on the students' year of study.

Cluster 3. There was a consensus among participants regarding experiment, creativity, communication and design learning objectives, as students indicated that their laboratory experiences had not offered any opportunities that promoted these learning objectives.

\subsection{Cluster 1}

Psychomotor and sensory awareness learning objectives relate to the manipulation of objects/apparatus (i.e., practical hands-on skills) [13], and the coordination between the brain and body in performing tasks [2]. When discussing psychomotor, the students commented that most of the techniques get repeated, and therefore, it gives them a chance to improve their skills. For example, titration was mentioned as a "reflex". One point worth mentioning is that the definition of psychomotor, which was given to the students, is "demonstrate competence in selection, modification, and operation of appropriate engineering tools and resources". Student responses mostly related to modification and operation rather than selection of tools and resources. This is not surprising, as they usually have to follow laboratory procedures.

Sensory awareness is defined as "use the human senses to gather information and to make sound engineering judgments in formulating conclusions about real-world problems". Students had positive opinions about sensory awareness, and indicated they were fairly confident in making decisions based on colour, temperature and pressure. In addition, they used words, such as "colour", "hot" and "pressure build up", which are related to the five traditional human senses. In general, most students in the focus groups, thought the laboratories had helped them achieve learning outcomes related to practical experience. This has been shown in other studies as well [10].

Another advantage of hands-on laboratories, especially compared to simulations, is that they demonstrate both the power and limitations of theories [4]. Students can learn to explain and reconcile the differences between theories and experimental results [4]. Simulations, on the other hands, cannot fully represent the complexity of real world systems and behaviors, as they essentially only represent mathematical models $[4,13]$. While discussing the models learning objective, students stated that they now understand reactions may not necessarily result in a $100 \%$ yield in contrast to what theories predict. One student explicitly commented "[the] secondary point of doing labs is to see how different it is from theory". Therefore, it seems that laboratories have a benefit in terms of helping students achieve the models learning objective.

\subsection{Cluster 2}

The teamwork learning objective depends mainly on how the laboratory course is designed. For example, thirdyear students indicated a greater teamwork learning experience since they performed experiments as a group compared to second-year students, who performed 
experiments individually. Teamwork is an important skill expected from engineering students [5]. However, individual work can also be beneficial as one student explicitly commented "[When working on your own], you learn a lot more. It is on you to make sure you understand everything you are doing". Therefore, a balance of individual work and teamwork in laboratories could be beneficial, as it helps students improve different skill sets.

The students had different opinions regarding safety. Second-year students had the impression that a huge focus of laboratories is on safety, whereas third- and fourth-year students indicated that if an accident happens, they would not immediately know how to handle it. Rather, they are relying on others; as one participant said, "at least someone in the team would [know what to do]". This transition from the second to third/fourth year may also reflect a natural maturation. In their second-year, students seem to be emphasizing what they are being taught, whereas in third year they are more aware of the limits in what they know. Nevertheless, the discussion was concerning, as safety is an important issue, regardless of it being a learning objective. Therefore, more emphasis should be placed on safety to make sure students are confident in case of accidents.

Instrumentation is defined as "apply appropriate sensors, instrumentation and /or software tools to make measurements of physical quantities". The definition could be divided into three parts: recognize the appropriate tool, know when to use it and know how to use it. Second-year students indicated that laboratories had helped them achieve this learning objective. However, it seemed that they interpreted the definition slightly differently as they mostly focused on performing techniques, such as crystallization or filtration, rather than on using instruments to make measurements. Only one student said "[I now know] why the UV [spectrophotometer] is used". Third- and fourth-year students, on the other hand, commented that they barely remember the purpose of the instruments. One participant said "we do what we are told", while another participant commented "In third year, it is very intense. You need more time to really appreciate all the instruments". Therefore, it seems that the instruments get more complicated in third-year laboratories, and students retain less of what they learn, which could partially be due to lack of repetition.

The discussion regarding ethics in the lab was interesting. Second-year students stated they cannot change the data, even if they want to, because they have to record their observations and results in their notebooks. In addition, laboratories usually include instruments, which generate spectral data that is unequivocal and accurate with respect to the analyte/analytes present. This was specifically discussed with respect to organic laboratories. On the other hand, a third-year student stated that sometimes they change their data, because the grades depend on them, and "there is too much pressure on getting good grades". Fourth-year students mentioned that at times, the TAs tell them to ignore their own data and use someone else's. However, this should be mentioned in their report and hence "it is not really an ethical problem". Other than that, they cannot change their data since they usually have to submit a copy of the raw data. Copying others' experimental data has been observed in other studies as well [10], which could mostly be because students are assessed based on their results/products. On the other hand, students should also learn that results actually do matter. Therefore, finding the right amount of emphasis on results seems to be the challenge.

When discussing learn from failure, second-year students said most of the techniques, especially in organic laboratories, get repeated and so "you can learn from previous laboratories". Third-year students, on the other hand, said they usually don't have time to repeat experiments even if they know what went wrong. Fourthyear students agreed that repeating the experiments to fix the mistakes depends on the type of laboratory and timing.

The discussion regarding data analysis was not clear. Third-year students said "[data analysis] is pretty much all we do", whereas second- and fourth-year students commented that they rarely do any data analysis. This is interesting as one main goal of performing laboratory experiments is to analyze and interpret data. Unfortunately, the limited time of the focus groups prevented obtaining more insight about this issue.

\subsection{Cluster 3}

Students are usually instructed to follow given laboratory procedures, and they are not provided with opportunities to be creative and develop experimental procedures independently. Therefore, it is not surprising that they said that the experiment and creativity learning objectives are missing in the laboratories. When discussing experiment, one student commented "that is a problem we don't usually get experiments that we get to have free will on how to design." Interestingly, however, students had different opinions when asked if they would like to devise their own experimental procedures. Secondand third-year students said they would like it only if they are given enough information, and know what they are doing. Fourth-year students, on the other hand, were more open to the idea, and even suggested that it should be included in the curriculum, as "it will give you a chance to show you can apply your lab skills". This could be due to increased maturity and increasing comfort with ambiguity for fourth-year students.

When discussing communication, most students stated that the reports were fairly simple and basic, which was true at the time of the focus group. However, the course instructors have recently introduced a few executive 
summaries and technical reports for some of the laboratories, which could help improve communication skills.

Design projects are essential requirements for most undergraduate engineering curricula, as they help students improve skills such as problem solving, communication, teamwork and leadership [16]. Most of the students reflected positively about their design experiences, due to connection to lectures/real life and attainment value. However, the discussion was in relation to design courses, rather than laboratory courses, as chemical engineering laboratory courses at the UofT usually do not involve design projects.

\section{Discussion}

A study was conduced by Most and Deisenroth (2006) [11], in which five laboratory instructors were asked to complete a survey by rating their courses' correlation and coverage according to the definitions of the learning objectives. Similar to our findings, the instructors also said that resource and time constraints usually prohibit opportunities for experiment and creativity, and suggested that design might not be a suitable learning objective for the laboratories. The instructors also had a few suggestions for the definitions, and showed concern about students being able to fully understand some of the words in the definitions. The students in our study were not specifically asked to comment on the clarity of the definitions. However, they didn't seem to have any major problems with the definitions either. Further studies can be done to determine how students interpret the definitions.

Some of the gaps mentioned by the students (and the instructors in [11]) can be addressed by exploiting the benefits of virtual and remote laboratories (VRLs). Virtual labs are computer software/models, which provide simulated data [13]. Remote laboratories, on the other hand, provide access to equipment via the Internet, and hence provide students with real data [13]. Combining VRLs with hands-on laboratories has been shown to increase the conceptual understanding of students in laboratories $[15,20,21]$. Simulations, in particular, provide a safe environment for performing experiments that are potentially dangerous in the real world [19]. In addition, they can be used to explore "what if" situations [18] by changing variables that are unrealistic or impossible to change in the real world [6]. VRLs also allow students to focus on experimental observations, rather than the physical manipulation of instruments or techniques [6]. In addition, students can conduct many more experiments easier, faster and cheaper compared to hands-on labs and repeat them as needed [6]. Therefore, VRLs can compliment hands-on laboratory experiments to provide opportunities for students to devise their own experiments, be creative without worrying about safety, and repeat them as necessary.

A change in the format of laboratory reports, as has already been initiated, could improve communication skills. In addition, instead of having students passively write a quiz about safety, other active learning approaches could be employed to teach students about it. This could potentially improve students' ability and confidence with regard to safety.

\section{Limitations and Future Studies}

One limitation of this study is the reliance on student perceptions regarding the definitions of learning objectives. Students might have interpreted some of the definitions differently than intended. In addition, the nature of qualitative studies does not allow for generalization to a larger group and other disciplines. Another limitation is time constraints. The discussion on learning objectives was just one of the topics covered in the focus group, and in some cases more questions could have been asked to clarify the discussion. Therefore, another focus group with the sole purpose of discussing learning objectives might result in a more complete picture. A focus group/interview with the instructors similar to the study by [11] could also be helpful to get more insight on the effectiveness of laboratories with respect to these learning objectives.

\section{Conclusion}

A focus group was conducted with undergraduate chemical engineering students at the University of Toronto (St. George campus) to gain insight into their perceptions regarding undergraduate laboratories. The thirteen laboratory learning objectives, developed at the ABET colloquy in 2002, were used as a framework to determine the strengths and limitations of laboratories. Based on student discussions, it seems that hands-on laboratories can help students improve practical skills (psychomotor and sensory awareness), and understand limitations of theories (models), while communication, ethics in the lab, and safety need improvements. Combining VRLs with hands-on laboratories can address the gaps mentioned by students relating to developing experimental procedures independently (experiment), creativity and repeating experiments as necessary to learn from mistakes (learn from failure). We hope to address some of the gaps identified in the focus groups through the development of digital learning objects. For example, we have already started to develop some web-based prelab modules to address some of the safety issues among other subjects such as theory and procedural steps. The development of simulations is also under way, to provide an opportunity for students to apply their knowledge to new problems, be creative and repeat experiments. 


\section{Acknowledgements}

This research is supported in part by the Ontario Graduate Scholarship, and the stipend from the Collaborative Engineering Education Program at UofT. The authors would also like to thank the students for their participation in the study.

\section{References}

[1] Avi Hofstein and Vincent N. Lunetta, "The laboratory in science education: Foundations for the twenty-first century." Science education, vol. 88, no. 1, pp. 28-54, 2004.

[2] A. R. M. Zaghloul, "Assessment of Lab Word: A threeDomain Model; Cognitive, Affective and Psychomotor." In Proc. American Society for Engineering Education Annual Conference \& Exposition, ASEE2001, (Albuquerque, New Mexico: 24-27 June 2001), 7 pp., 2001.

[3] Daniel S. Domin, "A review of laboratory instruction styles," Jounrtal of Chemical Education. Vol. 76, no. 4, p. 543,1999 .

[4] D. Magin and S. Kanapathipillai, "Engineering students' understanding of the role of experimentation," European journal of engineering education, vol. 25, no. 4, pp. 351$358,2000$.

[5] Ellen L. Walker and Oberta A. Slotterbeck, "Incorporating realistic teamwork into a small college software engineering curriculum," Journal of computing sciences in colleges, vol. 17, no. 6, pp. 115-123, 2002.

[6] Georgios Olympiou and Zacharias C. Zacharia, "Blending physical and virtual manipulatives: An effort to improve students' conceptual understanding through science laboratory experimentation," Science Education, vol. 96, no. 1, pp. 21-47, 2012.

[7] James E. Corter, Sven K. Esche, Constantin Chassapis, Jing $\mathrm{Ma}$, and Jeffery V. Nickerson. "Process and learning outcomes from remotely-operated, simulated, and hands-on student laboratories," Computers \& Education, vol. 57, no. 3, pp. 2054-2067, 2011.

[8] Jing Ma and Jeffery V. Nickerson, "Hands-on, simulated, and remote laboratories: A comparative literature review," ACM Computing Surveys (CSUR), vol. 38, no. 3, p. 7, 2006.

[9] John Dewey, Experience and Education. The Kappa delta Pi Lectures Series. London: Collier Mac Millan, 1963. 90 pp.

[10] Kamilah R. Salim, Habibah N. Haron, Noor H. Hussain, and Rosmah Ali, "The Achievement of Laboratory Work Learning outcomes: Students' Perceptions," in Proc. Research in Engineering Education Symposium, REES13, (Kuala Lumpur, Malaysia; 4-6 July, 2013 ), 6 pp., 2013.
[11] Karen R. Most, Michael P. Deisenroth, “ABET and Engineering Laboratory Learning Objectives: A Study at Virginia Tech," in Proc. American Society for Engineering Education Annual Conference \& Exposition, ASEE2003, (Nashville, TN; 22-25 June 2003), 20 pp., 2003.

[12] Lyle D. Feisel and George D. Peterson, "A colloquy on learning objectives for engineering education laboratories," in Proc. American Society for Engineering Education Annual Conference \& Exposition, ASEE2002, (Montreal, Canada; 16-19 June 2002), 13 pp., 2002.

[13] Lyle D. Feisel and Albert J. Rose, "The role of the laboratory in undergraduate engineering education," Engineering Education, vol. 94, no. 1, pp. 121-130, 2005.

[14] Mahmoud Abdulwahed and Zoltan K. Nagy, "Applying Kolb's experiential learning cycle for laboratory education," Engineering Education, vol. 98, no. 3, pp. 283-294, 2009.

[15] Mahmoud Abdulwahed and Zoltan K. Nagy, "The TriLab, a novel ICT based triple access mode laboratory education model," Computers \& Education, vol. 56, no. 1, pp. 262$274,2011$.

[16] Marcia K. Friesen, Lynn Taylor, and M. G. Britton, “A qualitative study of a course trilogy in biosystems engineering design," Journal of Engineering Education, vol. 94, no. 3, pp. 287-296, 2005.

[17] Romanas V. Krivickas and Jonas Krivickas, "Laboratory instruction in engineering education," Global J. Eng. Educ, vol. 11, no. 2, pp. 191-196, 2007.

[18] Sara Hennessy, Jocelyn Wishart, Denise Whitelock, Rosemary Deaney, Richard Brawn, Linda la Velle, Angela McFarlane, Kenneth Ruthven, Mark Winterbottom, "Pedagogical approaches for technology-integrated science teaching," Computers \& Education, vol. 48, no. 1, pp. 137-152, 2007.

[19] Yasser Elawady and A. S. Tolba, "Educational objectives of different laboratory types: A comparative study," International Journal of Computer Science and Information Security, vol. 6, no. 2, pp. 89-96, 2009.

[20] Zacharias C. Zacharia and Georgios Olympiou. "Physical versus virtual manipulative experimentation in physics learning," Learning and Instruction, vol. 21, no. 3, pp. 317-331, 2011.

[21] Zacharias C. Zacharia, "Comparing and combining real and virtual experimentation: an effort to enhance students' conceptual understanding of electric circuits," Journal of Computer Assisted Learning, vol. 23, no. 2, pp. 120-132, 2007. 


\section{APPENDIX A: Learning Objectives Definitions}

Objective 1: Instrumentation. Apply appropriate sensors, instrumentation, and/or software tools to make measurements of physical quantitates.

Objective 2: Models. Identify the strengths and limitations of theoretical models as predictors of real-world behaviors. This may include evaluating whether a theory adequately describes a physical event and establishing or validating a relationship between measured data and underlying physical principles.

Objective 3: Experiment. Devise an experimental approach, specify appropriate equipment and procedures, implement these procedures, and interpret the resulting data to characterize and engineering material, component, or system.

Objective 4: Data Analysis. Demonstrate the ability to collect, analyze, and interpret data, and to form and support conclusions. Make order of magnitude judgments and use measurement unit systems and conversions.

Objective 5: Design. Design, build, or assemble a part, product, or system, including using specific methodologies, equipment, or materials; meeting client requirements; developing system specifications from requirements; and testing and debugging a prototype, system, or process using appropriate tools to satisfy requirements.

Objective 6: Learn from Failure. Identify unsuccessful outcomes due to faulty equipment, parts, code, construction, process, or de- sign, and then re-engineer effective solutions.
Objective 7: Creativity. Demonstrate appropriate levels of in- dependent thought, creativity, and capability in realworld problem solving.

Objective 8: Psychomotor. Demonstrate competence in selection, modification, and operation of appropriate engineering tools and resources.

Objective 9: Safety. Identify health, safety, and environmental issues related to technological processes and activities, and deal with them responsibly.

Objective 10: Communication. Communicate effectively about laboratory work with a specific audience, both orally and in writing, at levels ranging from executive summaries to comprehensive technical reports.

Objective 11: Teamwork. Work effectively in teams, including structure individual and joint accountability; assign roles, responsibilities, and tasks; monitor progress; meet deadlines; and integrate individual contributions into a final deliverable.

Objective 12: Ethics in the Laboratory. Behave with highest ethical standards, including reporting information objectively and interacting with integrity.

Objective 13: Sensory Awareness. Use the human senses to gather information and to make sound engineering judgments in formulating conclusions about real-world problems. 